\title{
A role for exogenous GLP-1 in the management of postprandial hypoglycaemia after Roux-en-Y gastric bypass?
}

\section{Caroline C Øhrstrøm ${ }^{1}$ and Filip K Knop $2,3,4,5$}

${ }^{1}$ Department of Medicine, Zealand University Hospital, University of Copenhagen, Køge, Denmark, ${ }^{2}$ Center for Clinical Metabolic Research, Gentofte Hospital, University of Copenhagen, Hellerup, Denmark, ${ }^{3}$ Department of Clinical Medicine, Faculty of Health and Medical Sciences, University of Copenhagen, Copenhagen, Denmark, ${ }^{4}$ Novo Nordisk Foundation for Basic Metabolic Research, Faculty of Health and Medical Sciences, University of Copenhagen, Copenhagen, Denmark, and ${ }^{5}$ Steno Diabetes Center Copenhagen, Gentofte, Denmark
Correspondence should be addressed to C C Øhrstrøm or F K Knop Email

cacg@regionsjaelland.dk or filip.krag.knop.01@regionh. $\mathrm{dk}$

\begin{abstract}
Roux-en-Y gastric bypass (RYGB) is one of the most common and successful bariatric surgeries. However, more than half of RYGB-operated individuals may suffer from post-bariatric hypoglycaemia (PBH) characterised by traditional hypoglycaemic symptoms occurring 1 to 4 hours after meal intake. The mechanisms underlying PBH most likely relate to accelerated delivery of nutrients to the small intestine resulting in unretarded nutrient absorption, large elevations in postprandial plasma glucose concentrations (constituting a potent insulin secretory stimulus), and grossly elevated postprandial plasma levels of the insulinotropic gut-derived hormone glucagon-like peptide 1 (GLP-1) potentiating glucose-stimulated insulin secretion. Based on previous findings that circulating GLP-1 concentrations increased by $100 \%$ during insulin-induced hypoglycaemia before but not after RYGB, Almby et al. explored whether exogenous GLP-1 may protect against PBH. They performed hyperinsulinaemic hypoglycaemic clamps with concomitant infusion of the GLP-1 analogue exenatide and saline, respectively, in individuals who had undergone RYGB surgery. Infusion with exenatide during hypoglycaemia had no plasma glucose-raising effects, did not increase the counterregulatory glucagon response, and did not affect symptom scores. In the present commentary, potentially important implications derived from the study by Almby et al. published in the August issue of EJE, are discussed in the light of previous observations on GLP-1 receptor agonist treatment in $\mathrm{PBH}$. While the findings by Almby et al. do not provide a solution for patients with $\mathrm{PBH}$, they contribute to the knowledge base needed to address the growing problem of $\mathrm{PBH}$.
\end{abstract}

European Journal of Endocrinology (2019) 181, C5-C8

Roux-en-Y gastric bypass (RYGB) is one of the most common bariatric surgeries. The procedure induces a sustained weight loss together with a reduction in obesityrelated comorbidities including type 2 diabetes. The effects on postprandial glucose metabolism are evident within days or weeks after surgery - before any weight loss has occurred - and are thought to be achieved by a faster passage of nutrients to the small intestine and ensuing alterations in the secretion of several gastrointestinal hormones, acceleration of glucose absorption and increased insulin secretion (1). Especially the remarkable increase in postprandial plasma levels of the gut-derived incretin hormone glucagon-like peptide 1 (GLP-1) with not only a potent insulinotropic effect, but also a glucagonostatic effect - is believed to contribute to the metabolic improvements and the early remission of type 2 diabetes seen after RYGB (1).

Postprandial glycaemic excursions after RYGB are characterised by an early steep rise in plasma glucose to higher peak values than preoperatively, followed by a

Published by Bioscientifica Ltd. 
fast drop to baseline values (2). In some RYGB-operated individuals, the slope by which postprandial plasma glucose concentrations drop after peaking is very steep impeding a smooth reversion to baseline concentrations. In these individuals, plasma glucose concentrations may continue to drop below $3.9 \mathrm{mmol} / \mathrm{L}$, hereby resulting in postprandial hypoglycaemia. This post-surgical complication of RYGB is increasingly being referred to as post-bariatric hypoglycaemia $(\mathrm{PBH})$ and is characterised by traditional hypoglycaemic symptoms occurring $1-4 \mathrm{~h}$ after meal intake (3). The clinical suspicion of $\mathrm{PBH}$ can be verified by documenting postprandial hypoglycaemia during continuous glucose monitoring (CGM) or during a meal provocation test. The exact prevalence of $\mathrm{PBH}$ is unknown but using CGM, postprandial hypoglycaemia has been demonstrated to occur in up to $75 \%$ of RYGBoperated individuals, although many of the hypoglycaemic episodes appear to be asymptomatic $(4,5)$.

The mechanisms underlying $\mathrm{PBH}$ most likely relate to the altered intestinal anatomy, which as aforementioned leads to an accelerated delivery of nutrients to the small intestine resulting in unretarded nutrient absorption. The accompanying rapid and large elevations in postprandial plasma glucose concentrations constitute a potent insulin secretory stimulus at the level of the pancreatic beta cells. Further, the ingested nutrients come into immediate contact with the mucosa of the distal jejunum where GLP-1-secreting enteroendocrine cells are much more abundant compared with the duodenum (the normal entry point of ingested nutrients into the small intestine). This results in grossly elevated (up to 20-fold) circulating postprandial plasma levels of endogenous GLP-1 (2). As the insulinotropic effect of GLP-1 is glucose-dependent and increases exponentially with increasing plasma glucose concentrations, the rapid elevation of postprandial plasma glucose combined with the grossly elevated plasma GLP-1 concentrations unleash a dramatic insulin response from the pancreatic islets clearly exceeding the need. In individuals with $\mathrm{PBH}$, this insulin overshoot ultimately causes plasma glucose levels to decrease to hypoglycaemic levels $(6,7)$. The reason why not all RYGBoperated individuals develop $\mathrm{PBH}$ remains uncertain (3). Nonetheless, individuals with $\mathrm{PBH}$ appear to have higher postprandial GLP-1 and insulin levels compared with non-hypoglycaemic individuals, supporting the notion that an exaggerated GLP-1 response plays a role in the hypersecretion of insulin triggering $\operatorname{PBH}(8,9)$.

In a study published in the August issue of EJE, Almby et al. explored whether exogenous GLP-1 may protect against $\mathrm{PBH}$ (10). The group previously demonstrated that circulating GLP-1 concentrations increased by $\sim 100 \%$ during insulin-induced hypoglycaemia in 12 obese individuals without diabetes referred to RYGB (11). This observation was puzzling as no intraluminal nutrients or other known 'GLP-1 secretagogues' were introduced during the experiment. Preliminary results from our group support that hypoglycaemia can increase circulating levels of GLP-1 in man without administration of oral or intraluminal 'GLP-1 secretagogues' (M Christensen, L Vedtofte, JJ Holst, T Vilsbøll \& FK Knop, unpublished observations). The mechanisms behind this phenomenon remain obscure but may relate to a direct effect of hypoglycaemia on enteroendocrine GLP1-secreting cells or - perhaps more likely - constitute a by-product of the massive glucagon secretion from pancreatic alpha cells during hypoglycaemia (possibly due to unspecific processing of proglucagon). Interestingly, when the abovementioned 12 individuals were subjected to the same step-wise induction of hypoglycaemia 4-5 months after surgery, they not only observed attenuated counterregulatory responses of glucagon, epinephrine, norepinephrine and cortisol, the investigators also found a much smaller GLP-1 response during hypoglycaemia, when compared with pre-surgical conditions (in accordance with the notion of GLP-1 being co-secreted with glucagon during hypoglycaemia presented above) (11). Although GLP-1 is not traditionally considered an important counterregulatory hormone and is known to exert a potent suppressive effect on glucagon secretion from alpha cells at elevated plasma glucose concentrations, the authors speculated that increased GLP-1 levels during hypoglycaemia could stimulate the secretion of glucagon. These previous findings and speculations constituted the incentives for Almby et al. to perform the study reported in the present issue. In this study, they performed hyperinsulinaemic-hypoglycaemic clamps with concomitant infusion of the GLP-1 analogue exenatide $(0.066 \mathrm{pmol} / \mathrm{kg} / \mathrm{min})$ and saline, respectively, in 12 individuals (without history of type 1 or type 2 diabetes) who had undergone RYGB surgery 12 months prior to the study. The primary objective was to test whether exogenous GLP-1 would enhance counterregulatory hormonal responses and reduce hypoglycaemic symptoms. In brief, infusion with exenatide during hypoglycaemia had no plasma glucose-raising effects, did not increase the counterregulatory glucagon response and did not affect symptom scores (10).

Almby et al. are to be praised for their commitment to follow-up on their previous findings and, despite the negative nature of the results, potentially important 
implications with regard to previous observations on GLP-1 receptor agonist treatment in $\mathrm{PBH}$ may be derived from their study. The same group and others have reported reduced hypoglycaemic symptoms and improved glycaemic control in individuals with $\mathrm{PBH}$ during treatment with the GLP-1 receptor agonists liraglutide and exenatide when evaluated by CGM (9, 12). Considering the exaggerated postprandial GLP-1 response that supposedly contributes to $\mathrm{PBH}$ and the fact that GLP-1 antagonism has been shown to prevent postprandial hypoglycaemia in RYGB-operated individuals (8), this was a somewhat surprising finding, of which the possible mechanisms have been debated. As opposed to the acute postprandial elevation in endogenous GLP-1 concentrations, it has been suggested that continuous stimulation of GLP-1 receptors by exogenous GLP-1 (resulting in supraphysiological circulating GLP-1 receptor agonist concentrations) might have beneficial effects on postprandial hypoglycaemia and glycaemic variability (9). In the present study by Almby et al. the authors were not able to confirm their hypothesis that GLP-1 receptor agonism could reduce hypoglycaemia through an increased glucagon secretion in response to falling blood glucose concentrations. Our group recently examined the effects of 3-week treatment with the GLP-1 receptor agonist liraglutide in RYGB-operated individuals with $\mathrm{PBH}$, both during CGM and in an experimental setting (13). Following a provocative liquid test meal, we found no effects of liraglutide on postprandial hypoglycaemia, and treatment with liraglutide did not affect the number of hypoglycaemic events registered during CGM; however, our results suggested a reduction in CGM-verified time in the hyperglycaemic range and in parameters of glycaemic variability. Consistent with the known side effects of GLP-1 receptor agonists, we found that treatment with liraglutide was accompanied by complaints of nausea and reduced appetite, leading us to speculate that the glycaemic and symptomatic improvements reported during treatment could be the result of reduced food intake and altered food preferences, as especially carbohydrate restriction is known to diminish postprandial hypoglycaemia after RYGB (14). Unfortunately, dietary evaluation has not been performed in relation to treatment with GLP-1 receptor agonists in individuals with $\mathrm{PBH}$. The appetite-inhibiting effects of GLP-1 receptor agonists might also lead to weight loss, which is a desired effect by many individuals with $\mathrm{PBH}$ in whom weight regain is frequently observed (15). In other populations, GLP-1 receptor agonists - especially shortacting GLP-1 receptor agonists - reduce postprandial glycaemia by deceleration of gastric emptying and slowing of gastrointestinal transit time (16). Given the extensive rearrangements of the gastrointestinal tract after RYGB and the rapid passage of nutrients to the small intestine, therapeutic effects on 'pouch emptying' seem less likely in a RYGB-operated population, but this remains to be investigated. In that perspective, a short-acting GLP-1 receptor agonist, as used by Almby et al. might be a better choice of treatment (16).

The present findings by Almby et al. contribute to the knowledge on potential therapeutic effects of treatment with GLP-1 receptor agonists in RYGB-operated individuals, but the choice of study population limits the generalizability of the results. The included participants did not have a history of hypoglycaemic complaints and $\mathrm{PBH}$ was not evaluated by CGM or a provocative test meal prior to study inclusion. Using CGM, studies have shown that individuals with $\mathrm{PBH}$ experience repeated hypoglycaemic episodes on a weekly basis $(4,5,13)$, which might affect the observed counterregulatory responses since recurrent hypoglycaemia is known to attenuate counterregulation in other populations. Moreover, Almby et al. studied the effects of a continuous exenatide infusion in the fasting state. As PBH occurs postprandially and often is preceded by early postprandial hyperglycaemia, exenatide administered subcutaneously in connection with meal intake might have different effects on glucose metabolism in these individuals. Nevertheless, in the study alluded to above (13), we examined the effects of treatment with liraglutide in a postprandial setting in individuals with verified $\mathrm{PBH}$ and, in agreement with Almby et al. we found no increases in pancreatic glucagon levels in response to hypoglycaemia (CC Øhrstrøm, DL Hansen, UL Kielgast, JJ Holst \& D Worm, unpublished observations). Thus, the mechanisms by which GLP-1 receptor agonists may ameliorate hypoglycaemic symptoms and potentially stabilise glycaemic excursions in individuals with $\mathrm{PBH}$ remain unknown and convincing evidence that treatment with GLP-1 receptor agonists leads to clinically detectable improvements in hypoglycaemia is still missing.

Despite the growing awareness of $\mathrm{PBH}$, there are no established treatment recommendations for affected individuals besides dietary modifications. In agreement with previous studies, we recently showed that the alphaglucosidase inhibitor acarbose and the somatostatin analogue pasireotide effectively prevented postprandial hypoglycaemia in an experimental setting, but the effects were not necessarily applicable to daily living and the treatments were associated with notable side effects $(13,17,18)$. Other suggested therapeutics in 
the management of $\mathrm{PBH}$ involve diazoxide, calcium channel blockers, sodium-glucose cotransporter-2 inhibitors, glucagon and, as discussed above, GLP-1 receptor antagonists $(3,13)$. Given the few available treatment options and the severe consequences associated with $\mathrm{PBH}$, including the risk of syncope, seizures, motor vehicle accidents, and potentially, an increased rate of accidental deaths $(3,19)$, this area of research merits further investigations.

\section{Declaration of interest}

F K Knop is an Editorial Board Member of European Journal of Endocrinology. $\mathrm{F} \mathrm{K} \mathrm{Knop} \mathrm{was} \mathrm{not} \mathrm{involved} \mathrm{in} \mathrm{the} \mathrm{review} \mathrm{or} \mathrm{editorial} \mathrm{process} \mathrm{for} \mathrm{this} \mathrm{paper,}$ on which he is listed as an author. C C Øhrstrøm has nothing to disclose.

\section{Funding}

This work did not receive any specific grant from any funding agency in the public, commercial or not-for-profit sector.

\section{Author contribution statement}

С С $\varnothing$ and F K K drafted, edited and approved the manuscript.

\section{References}

1 Madsbad S, Dirksen C \& Holst JJ. Mechanisms of changes in glucose metabolism and bodyweight after bariatric surgery. Lancet: Diabetes and Endocrinology 20142 152-164. (https://doi.org/10.1016/S2213$8587(13) 70218-3)$

2 Jørgensen NB, Jacobsen SH, Dirksen C, Bojsen-Møller KN, Naver L, Hvolris L, Clausen TR, Wulff BS, Worm D, Lindqvist Hansen D et al. Acute and long-term effects of Roux-en-Y gastric bypass on glucose metabolism in subjects with Type 2 diabetes and normal glucose tolerance. American Journal of Physiology: Endocrinology and Metabolism 2012303 E122-E131. (https://doi.org/10.1152/ajpendo.00073.2012)

3 Salehi M, Vella A, McLaughlin T \& Patti ME. Hypoglycemia after gastric bypass surgery: current concepts and controversies. Journal of Clinical Endocrinology and Metabolism 2018103 2815-2826. (https:// doi.org/10.1210/jc.2018-00528)

4 Kefurt R, Langer FB, Schindler K, Shakeri-Leidenmühler S, Ludvik B \& Prager G. Hypoglycemia after Roux-en-Y gastric bypass: detection rates of continuous glucose monitoring (Cgm) versus mixed meal test. Surgery for Obesity and Related Diseases 201511 564-569. (https://doi.org/10.1016/j.soard.2014.11.003)

5 Abrahamsson N, Edén Engstrom B, Sundbom M \& Karlsson FA. Hypoglycemia in everyday life after gastric bypass and duodenal switch. European Journal of Endocrinology 2015173 91-100. (https:// doi.org/10.1530/EJE-14-0821)

6 Salehi M, Gastaldelli A \& D'Alessio DA. Altered islet function and insulin clearance cause hyperinsulinemia in gastric bypass patients With symptoms of postprandial hypoglycemia. Journal of Clinical Endocrinology and Metabolism 201499 2008-2017. (https://doi. org/10.1210/jc.2013-2686)
7 Goldfine AB, Mun EC, Devine E, Bernier R, Baz-Hecht M, Jones DB, Schneider BE, Holst JJ \& Patti ME. Patients with neuroglycopenia after gastric bypass surgery have exaggerated incretin and insulin secretory responses to a mixed meal. Journal of Clinical Endocrinology and Metabolism 200792 4678-4685. (https://doi.org/10.1210/ jc.2007-0918)

8 Salehi M, Gastaldelli A \& D'Alessio DA. Blockade of glucagonlike peptide 1 receptor corrects postprandial hypoglycemia after gastric bypass. Gastroenterology 2014146 669-680.e2. (https://doi. org/10.1053/j.gastro.2013.11.044)

9 Tharakan G, Behary P, Wewer Albrechtsen NJ, Chahal H, Kenkre J, Miras AD, Ahmed AR, Holst JJ, Bloom SR \& Tan T. Roles of increased glycaemic variability, GLP-1 and glucagon in hypoglycaemia after Roux-en-Y gastric bypass. European Journal of Endocrinology 2017177 455-464. (https://doi.org/10.1530/EJE-17-0446)

10 Almby KE, Abrahamsson N, Lundqvist MH, Hammar U, Thombare K, Panagiotou A, Karlsson FA, Sundbom M, Wiklund U \& Eriksson J. Effects of GLP-1 on counter-regulatory responses during hypoglycemia after GBP surgery. European Journal of Endocrinology 2019181 161-171. (https://doi.org/10.1530/EJE-19-0171)

11 Abrahamsson N, Börjesson JL, Sundbom M, Wiklund U, Karlsson FA \& Eriksson JW. Gastric bypass reduces symptoms and hormonal responses in hypoglycemia. Diabetes 201665 2667-2675. (https:// doi.org/10.2337/db16-0341)

12 Abrahamsson N, Engstrom BE, Sundbom M \& Karlsson FA. GLP1 analogs as treatment of postprandial hypoglycemia following gastric bypass surgery: a potential new indication? European Journal of Endocrinology 2013169 885-889. (https://doi. org/10.1530/EJE-13-0504)

13 Øhrstrøm CC, Worm D, Højager A, Andersen D, Holst JJ, Kielgast UL \& Hansen DL. Postprandial hypoglycaemia after Roux-en-Y gastric bypass and the effects of acarbose, sitagliptin, verapamil, liraglutide and pasireotide. Diabetes, Obesity and Metabolism 2019 [epub]. (https://doi.org/10.1111/dom.13796)

14 Kellogg TA, Bantle JP, Leslie DB, Redmond JB, Slusarek B, Swan T, Buchwald H \& Ikramuddin S. Postgastric bypass hyperinsulinemic hypoglycemia syndrome: characterization and response to a modified diet. Surgery for Obesity and Related Diseases 20084 492-499. (https://doi.org/10.1016/j.soard.2008.05.005)

15 Varma S, Clark JM, Schweitzer M, Magnuson T, Brown TT \& Lee CJ. Weight regain in patients with symptoms of post-bariatric surgery hypoglycemia. Surgery for Obesity and Related Diseases 201713 1728-1734. (https://doi.org/10.1016/j.soard.2017.06.004)

16 Andersen A, Lund A, Knop FK \& Vilsbøll T. Glucagon-like peptide 1 in health and disease. Nature Reviews: Endocrinology 201814 390-403. (https://doi.org/10.1038/s41574-018-0016-2)

17 Valderas JP, Ahuad J, Rubio L, Escalona M, Pollak F \& Maiz A. Acarbose improves hypoglycaemia following gastric bypass surgery without increasing glucagon-like peptide 1 levels. Obesity Surgery 201222 582-586. (https://doi.org/10.1007/s11695-011-0581-0)

18 Tack J, Aberle J, Arts J, Laville M, Oppert JM, Bender G, Bhoyrul S, McLaughlin T, Yoshikawa T, Vella A et al. Safety and efficacy of pasireotide in dumping syndrome - results from a phase 2, multicentre study. Alimentary Pharmacology and Therapeutics 201847 1661-1672. (https://doi.org/10.1111/apt.14664)

19 Marsk R, Jonas E, Rasmussen F \& Näslund E. Nationwide cohort study of post-gastric bypass hypoglycaemia including 5,040 patients undergoing surgery for obesity in 1986-2006 in Sweden. Diabetologia 201053 2307-2311. (https://doi.org/10.1007/s00125010-1798-5)

Received 27 June 2019

Accepted 10 July 2019 